\title{
Universiteit
}

Leiden

The Netherlands

\section{Diffusion of vacancies in metal surfaces: theory and experiment,}

Saarloos, W. van; Gastel, R. van; Frenken, J.W.M.; Schwartzentruber, B.S.; Somfai, E.

\section{Citation}

Saarloos, W. van, Gastel, R. van, Frenken, J. W. M., Schwartzentruber, B. S., \& Somfai, E. (2003). Diffusion of vacancies in metal surfaces: theory and experiment,. The Chemical Physics Of Solid Surfaces, Vol.11 Surface Dynamics, Ed. D.p. Woodruff „, 351-370. Retrieved from https://hdl.handle.net/1887/5531

Version: $\quad$ Not Applicable (or Unknown)

License: $\quad$ Leiden University Non-exclusive license

Downloaded from: $\quad$ https://hdl.handle.net/1887/5531

Note: To cite this publication please use the final published version (if applicable). 
(C) 2003 Elsevier Science B.V. All rights reserved.

Surface Dynamics

D.P. Woodruff (Editor)

Chapter 12

\title{
Diffusion of vacancies in metal surfaces: theory and experiment
}

\author{
R. van Gastel ${ }^{a}$, J.W.M. Frenken ${ }^{a}$, B.S. Swartzentruber ${ }^{c}$, E. Somfai ${ }^{b}$ and W. van Saarloos ${ }^{b}$ \\ ${ }^{\text {a }}$ Universiteit Leiden, Kamerlingh Onnes Laboratory, PO Box 9504, 2300 RA Leiden, \\ The Netherlands \\ b Universiteit Leiden, Instituut-Lorentz, PO Box 9506, 2300 RA Leiden, The Netherlands \\ ${ }^{c}$ Sandia National Laboratories, Surface and Interface Sciences Department, P.O. Box \\ 5800, Mail Stop 1415, Albuquerque, NM 87185-1415, USA
}

\begin{abstract}
We review measurements and theory of vacancy-mediated mass transport in a metal surface. Experimentally, this transport is made visible with the use of a low density of tracer atoms, embedded in the outermost layer. The STM measurements presented in this chapter have been obtained with In and Pd atoms in the $\mathrm{Cu}(0 \mathrm{O} 1)$ surface. The observations show that the diffusion of these tracer atoms through the $\mathrm{Cu}(001)$ surface proceeds via long jumps separated by long time intervals. Quantitative details are discussed for both systems and we introduce the theoretical framework that was developed to analyze these measurements. Differences in the energetics of the vacancy-mediated diffusion of In and Pd are discussed and the mobility of $\mathrm{Cu}$ atoms in $\mathrm{Cu}(001)$ terraces is evaluated using these measurements.
\end{abstract}

\section{INTRODUCTION}

\subsection{The role of vacancies in surface diffusion}

The roughening and smoothing of a surface is governed by a combination of numerous atomic-scale processes, many of them involving the motion of single adatoms and vacancies. Over the years, images and movies of these elementary, atomistic diffusion processes on single crystal surfaces have been obtained with two key scientific instruments. The first of these, the Field Ion Microscope (FIM), has delivered real-space atomic scale images of small surface areas as early as the 1950s [1]. Over a period of several decades it has been the sole technique providing direct insight into the details of single adatom diffusion on most of the low-index transition metal surfaces [2, 3]. The Nobel Prize winning invention of the scanning tunneling microscope (STM) [4] has led to a renaissance of the field of surface diffusion in the 1980s. Ever since, the field has flourished and a great number of investigations have been performed to measure the elementary processes that are involved in diffusion on a wide range of surfaces under a wide range of conditions [5-7]. Over the past 20 years this has led to the establishment 
of an extensive knowledge base on the behavior of single crystal surfaces and the processes that govern mass transport across these surfaces.

The process of mass transport across a surface is usually thought to involve several stages. The first of these is the creation of a diffusing particle that is responsible for the mass transport. This is typically either an adatom (a single atom on top of the outermost atomic layer) or a surface vacancy. After their formation, these particles diffuse along steps or across terraces and they are typically incorporated again at a different site from the one where they were formed. This scenario obviously places a lot of emphasis on the role of steps, since it is typically at the steps where the diffusing particles are created. A great number of studies have focused on the dynamics of steps [8-15]. Even without observing the actual atomic processes, from the dynamics of steps and islands it can be established which atomic processes (e.g. diffusion along the step or terrace diffusion) are dominant. Combined with measurements of the adatom diffusion barriers on terraces $[2,16]$, and site-dependent measurements of local diffusion barriers like the diffusion barrier to cross a step [17-19], these studies provide a comprehensive picture of surface diffusion.

This picture may give the false impression that the only locations where surface atoms can be rearranged are at steps and kinks. Recently a few STM studies have been performed that shift the emphasis away from steps and focus instead on the large terraces that make up most of the surface [20-24]. A surface vacancy that is formed at one step and annihilated at another does not just cause a net displacement of an atom from the annihilation site to the birth site of the vacancy. As the vacancy travels through the terrace, it rearranges all surface atoms on its path. For elemental single crystal surfaces this diffusive motion is normally hidden from observation by the fact that all surface atoms are identical. However, through the use of a very dilute surface alloy, this diffusive motion can be directly visualized. More evidence for the role that vacancies play in mass transport across a surface has come from recent low energy electron microscopy (LEEM) studies [25, 26] which showed that the diffusion of vacancies, responsible for substantial surface rearrangements, is not necessarily confined to the surface layer of the crystal. In this chapter, we review recent studies of surface diffusion mediated by vacancies. We shall limit ourselves to those vacancies that form, diffuse, and annihilate exclusively in the surface layer.

\subsection{Can surface vacancies be important?}

As described above, the net rearrangement of atoms along the path of a diffusing vacancy within the terrace seems to be zero, since the configuration that is left behind looks identical to the one before the passage of the vacancy. Thus, one might be tempted to think that it is safe to ignore the vacancyinduced diffusion mechanism altogether. When small amounts of impurities are introduced, however, the dynamic nature of the terrace atoms becomes evident. In fact, in the examples that we shall review in this chapter the vacancy-mediated diffusion mechanism is the dominant mechanism in the transport of foreign atoms across a surface.

A seemingly strong argument in favor of the more traditional picture of surface diffusion is that on most surfaces the formation energy of surface vacancies is too high for them to be present in significant numbers and hence be of any importance. Taking the $\mathrm{Cu}\left(\begin{array}{ll}0 & 0\end{array}\right)$ surface as an example, using first-principles calculations, the formation energy of a vacancy was estimated to be $474 \mathrm{meV}$ [24]. At room temperature this corresponds to an equilibrium density of surface vacancies of a mere $7 \times 10^{-9}$. It should be noted that a similar situation exists for adatoms, with the estimated formation energy of an adatom being $507 \mathrm{meV}$ [27]. It is a common pitfall to think that the low density of vacancies automatically implies that their effect is negligible. The net effect of the vacancy diffusion mechanism is not solely determined by the vacancy formation energy. In fact, even at room temperature, the surface vacancies can have a significant effect on the diffusion of atoms in the terraces, when their diffusion barrier is sufficiently low. Again taking the example of $\mathrm{Cu}(001)$, using calculations based on the embedded atom method (EAM), we estimate the diffusion barrier for surface vacancies to be $350 \mathrm{meV}$ [28]. Assuming an attempt frequency of $10^{12} \mathrm{~Hz}$, this energy gives a vacancy hop rate of $1.3 \mathrm{MHz}$ at room temperature. The product of this high number and the low vacancy density estimated above, of 
approximately $10^{-2} \mathrm{~Hz}$ is the frequency with which we estimate each site on the surface to be visited by a vacancy at room temperature. In other words, despite the fact that there are so few vacancies at room temperature, their effect is still significant because their motion through the surface is extremely facile. Since the effect depends on the product of the density and the hopping rate, the relevant effective energy is the sum of the high formation energy and the low diffusion barrier. If we compare our estimate of

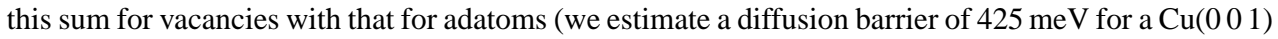
adatom [27]), we see that for this particular surface one should expect the net effect of vacancies to be significantly larger than that of adatoms.

\subsection{Can we see surface vacancy diffusion directly?}

From the numbers that are mentioned above it is obvious that the direct observation of surface vacancies is a formidable, if not an impossible, experimental challenge. The very combination of a low density and a high mobility makes the vacancy diffusion mechanism practically impossible to observe at room temperature. No technique available to date is capable of atomically resolving the structure of a surface with a temporal resolution better than a microsecond, as would be required by the diffusion rate calculated above. Also, no technique exists that can atomically resolve the structure of a surface in real space, on such a large length scale that the observation of significant numbers of naturally occurring vacancies would be feasible.

Two alternative approaches exist. The first one involves significantly lowering the temperature to values where the diffusion of vacancies can be observed with a technique like STM. At lower temperatures a surface vacancy can then be artificially created by ion bombardment or direct removal of an atom by the tip. This approach has been applied successfully to several semiconductor surfaces [29-31]. For metal surfaces, although vacancy creation at a step by direct tip manipulation of the surface has been demonstrated [32], to our knowledge, no studies have been published where the diffusion of artificially created vacancies in a terrace has successfully been measured. The second approach involves the addition of small amounts of appropriate impurities that serve as tracer atoms in the first layer of the surface [20-24]. The presence and passage of a surface vacancy is indirectly revealed by the motion of these embedded atoms. If one seeks to measure both the formation energy and the diffusion barrier of surface vacancies explicitly, a combination of these two approaches is needed.

In the remainder of this chapter we review measurements that were based on the dilute surface alloy approach for two different systems. We first discuss experimental results of the $\operatorname{In} / \mathrm{Cu}\left(\begin{array}{ll}0 & 0\end{array}\right)$ [21-23] and $\mathrm{Pd} / \mathrm{Cu}\left(\begin{array}{ll}0 & 0\end{array}\right)$ [24] systems. After this we discuss the theoretical framework that was developed to analyze the diffusion measurements [33]. We then discuss how to use this framework to interpret the measurements and discuss the differences between these two material systems. We also discuss the effect of steps on our measurements. Finally, in Section 6, we put the measurements in a broader context and discuss the relevance of these results for surfaces in general.

\section{STM MEASUREMENTS OF VACANCY-INDUCED DIFFUSION}

\subsection{Qualitative observations of vacancy-induced surface diffusion}

The first qualitative observation of vacancy-induced motion of embedded atoms was published in 1997 by Flores et al. [20]. Using STM, an unusual, low mobility of embedded $\mathrm{Mn}$ atoms in $\mathrm{Cu}(001)$ was observed. Flores et al. argued that this could only be consistent with a vacancy-mediated diffusion mechanism. Upper and lower limits for the jump rate were established in the low-coverage limit and reasonable agreement was obtained between the experimentally observed diffusion coefficient and a theoretical estimate based on vacancy-mediated diffusion. That same year it was proposed that the diffusion of vacancies is the dominant mechanism in the decay of adatom islands on $\mathrm{Cu}(001)$ [36], which was also backed up by ab initio calculations [37]. After that, studies were performed on the vacancy-mediated diffusion of embedded In atoms [21-23] and Pd atoms [24] in the same surface. The deployment of a high-speed variable temperature STM in the case of embedded In and an atomtracker STM in the case of Pd, allowed for a detailed quantitative investigation of the vacancy-mediated 
diffusion process by examining in detail both the jump frequency as well as the displacement statistics. Experimental details of both setups have been published elsewhere [34, 35]. A review of the quantitative results from these studies is presented in the next subsections.

\subsection{Direct proof for the role of vacancies}

We start the review of our quantitative observations by properly establishing that the diffusion of both embedded In and embedded $\mathrm{Pd}$ in $\mathrm{Cu}\left(\begin{array}{ll}0 & 0\end{array}\right)$ is driven by surface vacancies. One of the proofs, for the case of $\mathrm{In}$, is to be found in the incorporation process of the indium atoms. Figure 1 shows the $\mathrm{Cu}\left(\begin{array}{lll}0 & 0 & 1\end{array}\right)$ surface 38 minutes after the deposition of $0.03 \mathrm{ML}$ of indium at room temperature. The image shows a high density of indium atoms in the area around a step. STM images measured with bias voltages ranging from -0.10 to $-2.18 \mathrm{~V}$ always showed the indium atoms as bright protrusions with an approximate height of $0.4 \AA$. Comparison of the apparent height of the indium atoms observed in this image with that of indium adatoms that were observed in a separate experiment on a $\mathrm{Cu}\left(\begin{array}{lll}1 & 1 & 17\end{array}\right)$ surface $(\approx 2.55 \AA)$ [38], confirms that the indium atoms in Fig. 1 are indeed embedded in the first layer of the crystal. From the image it is obvious that the indium atoms have been incorporated in the surface through steps. The terraces are not populated uniformly by indium. The indium is found only in the direct vicinity of the steps. After deposition, once the indium adatoms have reached a step and attached themselves to it, they enter the first layer on both sides of the step, after which they spread uniformly throughout the first layer. At room temperature it takes the indium atoms typically several hours to spread homogeneously through the entire surface.

The spreading of the indium atoms through the first layer implies that they are able to diffuse, whilst remaining embedded within the surface. The diffusion behavior of the embedded indium atoms was studied in series of images of selected areas on the copper surface, forming STM movies of the motion.

The diffusion behavior of the indium atoms in these movies differs from the usual hopping mechanism in several respects, as is illustrated by the STM images in Fig. 2.

- The jumps of the indium atoms are separated by very long time intervals, which implies a rather high effective activation energy. At room temperature these intervals can be as long as a few minutes.

- If the indium atoms move between two images, they typically move over several atomic spacings, in contrast to single, monatomic hops.

- Neighboring indium atoms show a strong tendency to make their jumps simultaneously. If the indium atoms move independently from one another, they should not exhibit such concerted motion.

To explain the unusual diffusion behavior we invoke the existence of an assisting particle. First of all, as the assisting particle is invisible in the STM-images we have to assume that it is too mobile to be imaged with the STM. Second, if the assisting particle is present in very low numbers only, this could

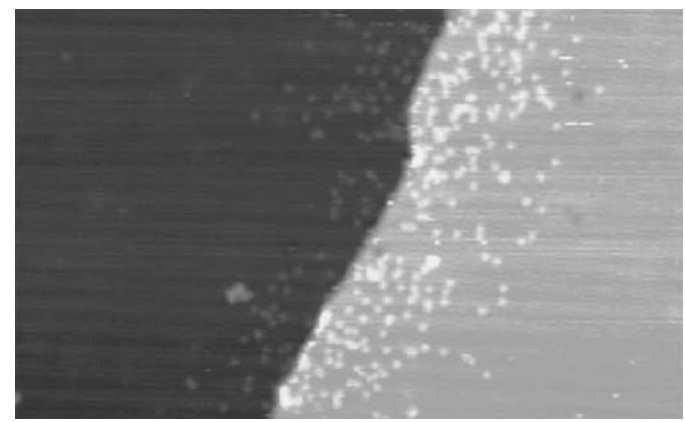

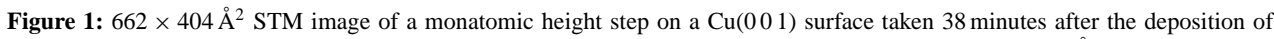
$0.03 \mathrm{ML}$ of indium at room temperature. Embedded indium atoms show up as bright dots with a height of $0.4 \AA$. The image shows a high density of embedded indium atoms near the step $\left(V_{t}=-1.16 \mathrm{~V}, I_{t}=0.1 \mathrm{nA}\right)$. 


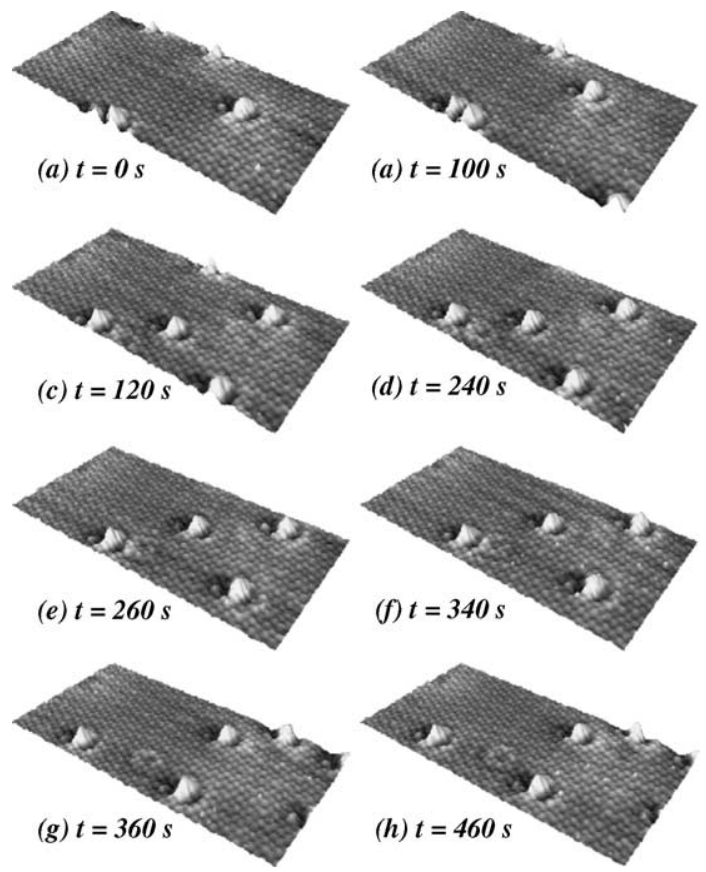

Figure 2: $140 \times 70 \AA^{2}$ STM images of the $\mathrm{Cu}(001)$ surface at room temperature illustrating the diffusion of embedded indium atoms. (a) shows five embedded indium atoms. The right hand panel (b) shows that after $100 \mathrm{~s}$ the indium atoms still occupy the same lattice sites. (c) shows the next image in which all indium atoms have made a multi-lattice-spacing jump. After this jump the indium atoms again stay at the same lattice site for another two minutes, illustrated by (d). (e-h) show that this pattern of long jumps separated by long time intervals repeats itself $\left(V_{t}=-0.58 \mathrm{~V}, I_{t}=0.9 \mathrm{nA}\right)$.

account for the long waiting times. Third, a multiple encounter between the assisting particle and the indium atoms may lead to a jump over several atomic spacings. And finally, if the assisting particle helps to displace one indium atom, there is a high probability that nearby indium atoms will also be displaced, leading to a concerted motion of the indium atoms.

We now consider the following two possibilities:

- Diffusion of embedded atoms through exchange with copper adatoms.

- Diffusion of embedded atoms through exchange with surface vacancies.

For exchange with a copper adatom the diffusion mechanism is illustrated in Fig. 3. In the measurements, embedded indium atoms are observed to make jumps of several atomic spacings. For the adatom mechanism, this would imply that the indium adatoms reinsert themselves into the terrace after making at most a few hops. From the room temperature deposition experiment in which the indium is deposited uniformly over the surface but only inserts into the terraces at a step, as shown in Fig. 1, we can exclude this mechanism. The adatom-exchange mechanism would lead to a uniform distribution of embedded indium atoms directly after deposition. By contrast, Fig. 1 clearly shows a high density of embedded indium atoms near a step shortly after deposition. The copper adatom-exchange mechanism can therefore be ruled out with confidence. We identify the exchange with surface vacancies as the mechanism responsible for the observed diffusion of indium through the surface. Figure 4 illustrates the vacancy-mediated diffusion mechanism.

The case of Pd is markedly different from that of indium in the sense that upon deposition the Pd atoms are immediately incorporated in the surface as opposed to attaching at the steps. This immediately 
(a)

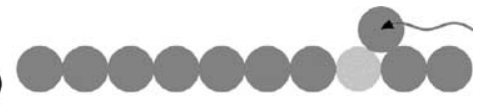

(b)

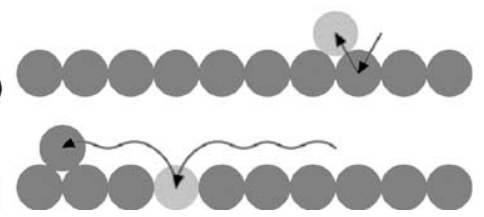

Figure 3: A cross-sectional view of an exchange process of indium (bright) with a copper (dark) adatom, leading to a long jump of the indium atom. (a) A copper adatom arrives at the embedded indium site, either through normal hopping or exchange hopping (not shown). (b) The copper adatom changes places with the indium atom. (c) The indium adatom now makes one or more hops over the surface before it reinserts itself into the first layer. A multiple encounter between the copper adatom and the indium may lead to even larger displacements.

yields a uniform distribution of embedded Pd atoms. The argument that was used above to establish the vacancy-mediated diffusion mechanism of In atoms, cannot be used for the case of Pd. Instead, the vacancy-mediated diffusion mechanism is distinguished from the adatom-exchange mechanism through an analysis of the distribution of jump lengths (shown in Fig. 6). In short, we consider the fraction of jumps of length $\sqrt{2}$ (expressed in atomic spacings). Since the Pd atoms incorporate into the terraces immediately upon deposition, the barrier to incorporate must be comparable to the diffusion barrier. Therefore, an embedded $\mathrm{Pd}$ atom that exchanges with a $\mathrm{Cu}$ adatom will not diffuse far before reinserting itself into the surface layer. In the case where the Pd atoms would immediately reinsert, the number of $\sqrt{2}$ jumps should be half that of single-length jumps. However, for vacancy-mediated diffusion, the fraction of $\sqrt{2}$ jumps is much lower because the vacancy must execute a minimum of four coordinated jumps in order to move the Pd diagonally. In the experiments, the ratio of $\sqrt{2}$ to single jumps is about 0.23 , much lower than the estimate for the adatom-exchange mechanism. The vacancy-mediated diffusion mechanism must therefore be active.

\subsection{Statistics of vacancy-induced diffusion}

One of the aspects associated with vacancy-mediated diffusion that differentiates it from the hopping mechanism on this surface is the long waiting time between consecutive jumps. An example of the distribution of waiting times has been plotted for both In and Pd in Fig. 5.

As can be seen from the figure, both distributions are purely exponential. The exponential shape of the distributions shows that the waiting time of an embedded atom is governed by a Poisson process with rate $\tau^{-1}$. This implies that subsequent long jumps are independent, which we take as proof that they are caused by different vacancies. The vacancies are created independently at random time intervals. The frequency with which a specific lattice site of the $\mathrm{Cu}(001)$ surface is visited by a new

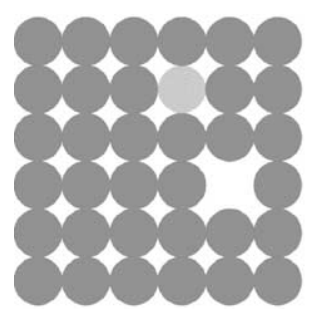

(a)

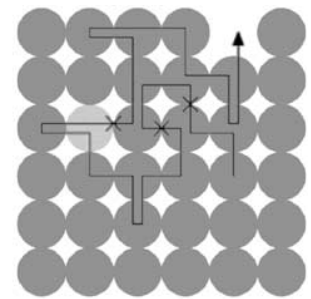

(b)

Figure 4: A ball-model (top view) of a diffusion event in which the passage of a surface vacancy leads to a multi-lattice-spacing displacement of the indium atom (bright). The arrow indicates the random walk pathway of the vacancy, and the indium-vacancy exchanges are marked with crosses to show the pathway of the indium between its beginning and endpoints. 


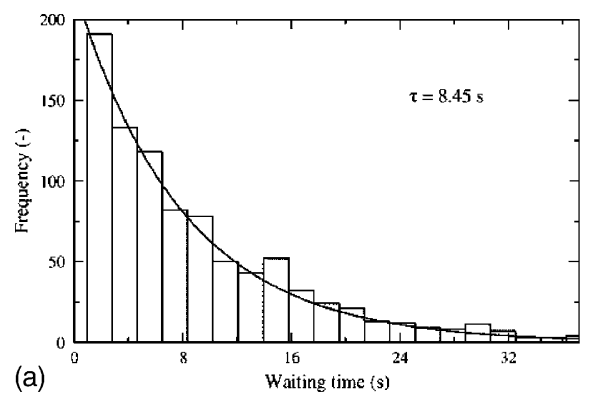

(a)

Figure 5: Distributions of waiting times between subsequent jumps in $\mathrm{Cu}(001)$, measured for (a) In at $320 \mathrm{~K}$ and (b) Pd at $335 \mathrm{~K}$. Both fits are pure exponentials. The time constant $t$ is shown in the graphs for both distributions.

vacancy is $\tau^{-1}$. This frequency is such that the diffusion process can easily be followed with both the high-speed STM and the atom-tracker STM up to a temperature of approximately $350 \mathrm{~K}$.

The diffusion of the embedded atoms in the surface proceeds through multi-lattice-spacing jumps separated by long time intervals. The multi-lattice-spacing nature of the diffusion is illustrated by the jump length distributions which are plotted in Fig. 6.

In Section 3 we derive that for the vacancy-mediated diffusion mechanism, one expects the shape of the jump length distribution to be that of a modified Bessel function of order zero. Both distributions can be fit very well with the modified Bessel function, again confirming the vacancy-mediated diffusion mechanism for both cases. The only free parameter used in the fits is the probability $\hat{p}_{\text {rec }}$ for vacancies to recombine at steps, between subsequent encounters with the same embedded atom [33]. This probability is directly related to the average terrace width and variations in this number can be ascribed to the proximity of steps. The effect of steps will be discussed in more detail in Section 4.

The jump length distribution shows that for the case of indium there is a significant probability for the embedded atoms to jump as far as five or six atomic spacings. In terms of the vacancy-mediated diffusion mechanism, if the vacancy were making an ordinary random walk and were not influenced by the presence of the indium, standard random walk theory [39] gives that the vacancy and the embedded atom must change places as often as twenty to thirty times to give such a large displacement. In the next section we will elaborate on how the presence of the In and Pd tracers affects the statistics of the vacancy and tracer diffusion process.
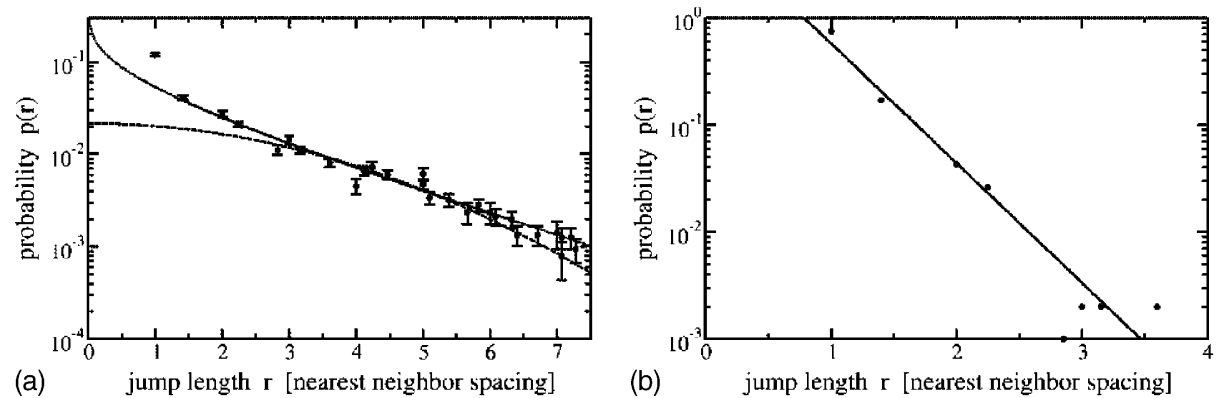

Figure 6: The distribution of jump lengths of embedded atoms in $\mathrm{Cu}(00$ ) $)$, measured for (a) $1461 \mathrm{In}$ hops at $320 \mathrm{~K}$ and (b) $887 \mathrm{Pd}$ hops at $335 \mathrm{~K}$. Plotted is the probability for a jump of an $\mathrm{In} / \mathrm{Pd}$ atom from its starting position to any site at a given distance from the starting position. Probabilities have been normalized so that the probabilities for the entire lattice add up to one. To illustrate the distinct nature of the diffusion behavior, the Gaussian jump length distribution that would be expected for the case of simple hopping is also plotted in (a). 


\section{THEORY OF TWO-DIMENSIONAL VACANCY-INDUCED TRACER DIFFUSION}

\subsection{Tracer diffusion on an infinite surface}

The problem of vacancy-mediated tracer diffusion in two dimensions has been investigated for a long time [40-44] and several different methods (simulation, analytical models, enumeration of trajectories, etc.) can be used to address it. The mathematics of this type of diffusion was solved first for the simplest case [41], when the diffusion of the vacancy is unbiased (all diffusion barriers are equal; the tracer atom is identical to the other atoms), the lattice is two-dimensional and infinite. There is a single vacancy present that makes a nearest-neighbor move in a random direction at regular time intervals and has an infinite lifetime, as there are no traps. The solution is constructed by separating the motion of the tracer and that of the vacancy. The correlation between the moves of the tracer atom is calculated from the probability that the vacancy returns to the tracer from a direction, which is equal, perpendicular or opposite to its previous departure. The probability density distribution of the tracer atom spreads with time, and on an infinite lattice for large times it approaches the following functional form:

$$
P_{t}(\boldsymbol{r})=\frac{2(\pi-1)}{\log t} K_{0}\left(\frac{r}{[\log t /(4 \pi(\pi-1))]^{1 / 2}}\right)
$$

where $K_{0}$ is the modified Bessel function of order zero [45] and time $t$ measures the number of moves of the vacancy. At $t=0$ the vacancy is next to the tracer atom. The non-Gaussian shape of this spatial distribution function is a signature of vacancy-mediated diffusion. The average displacement of the tracer particle diverges with time. This is a direct consequence of the fact that 2 is the marginal dimension for the return probability in the random walk problem. For higher dimensional problems, the probability that the vacancy returns to the tracer particle is less than unity and the average displacement of the tracer particle remains finite. However, for dimensions equal to or smaller than 2, the vacancy always returns to the tracer particle and as a consequence its displacement diverges.

The same problem has been solved in an alternate way for all dimensions [42]. From this solution one can calculate the number of tracer-vacancy exchanges up to time $t$. In two dimensions the distribution is geometric, with mean $(\log t) / \pi$. The continuum version of this problem has been considered as well in the form of an infinite-order perturbation theory [43]; the solution matches the asymptotic form of the lattice model.

In a very recent study the lattice calculations have been generalized to biased diffusion [44]. The difference between the tracer atom and the substrate atoms was taken into account by having different vacancy-tracer and vacancy-substrate exchange probabilities, while the rate of vacancy moves was kept constant. A repulsive interaction reduces, while a moderately attractive interaction increases the spreading of the tracer distribution.

Although these exact solutions are closely related to the two systems that we discuss here, the differences, e.g. in boundary conditions and vacancy lifetime, make a direct comparison with experiments impossible. For this purpose we develop a model of tracer diffusion, which includes the essential properties of the experimental system.

\subsection{Discrete model for tracer diffusion on a finite surface}

In this subsection we describe a discrete model for vacancy-mediated diffusion of embedded atoms, solve it numerically for the case of $\mathrm{In} / \mathrm{Cu}(001)$, and present the results. Our model is defined on a two-dimensional simple square lattice of size $l \times l$ (typically, $l=401$ ) centered around the origin. This corresponds to the top layer of a terrace of the $\mathrm{Cu}\left(\begin{array}{ll}0 & 0\end{array}\right)$ surface, with borders representing steps. The role of steps in the creation/annihilation of vacancies will be discussed in more detail in the next section. All sites but two are occupied by substrate atoms. At zero time the two remaining sites are the impurity (or tracer) atom, located at the origin, and a vacancy at position $(1,0)$. This corresponds to the situation immediately after the impurity atom has changed places with the vacancy.

The only allowed motion is the exchange of the vacancy with one of its neighboring atoms. The exchange rate depends on the local environment, i.e. on the relative position of the vacancy and the 


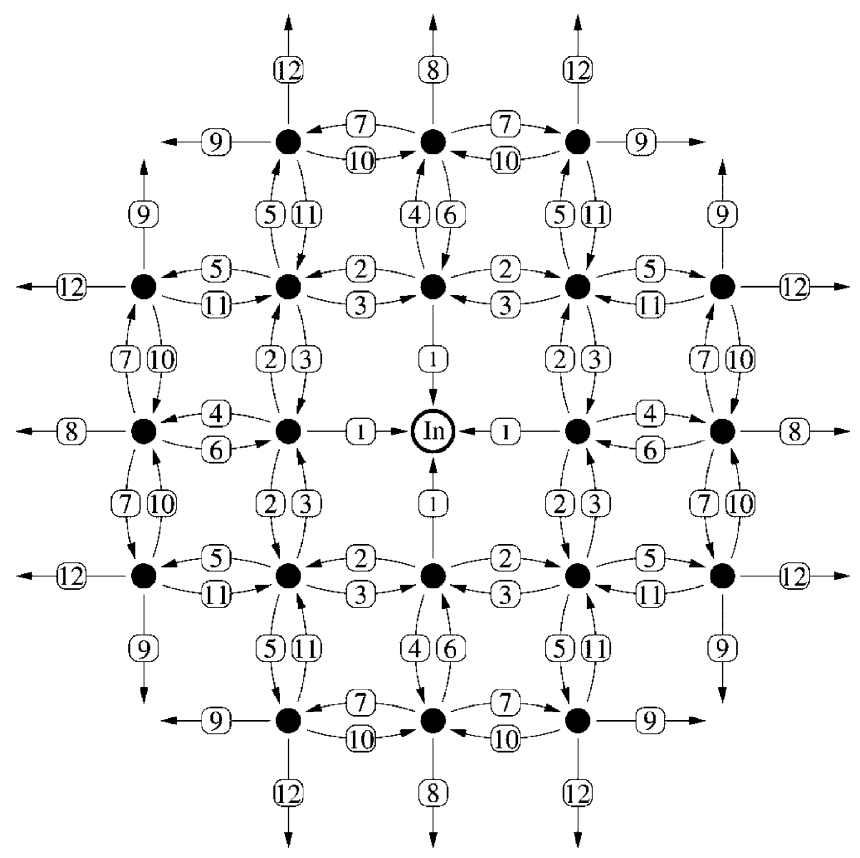

Figure 7: Special vacancy diffusion barriers near an In atom, calculated with the EAM method. The arrows denote the motion of the vacancy. The following values for the barriers were used in the calculation: $E_{1}=243 \mathrm{meV}, E_{2}=671 \mathrm{meV}, E_{3}=503 \mathrm{meV}$, $E_{4}=529 \mathrm{meV}, E_{5}=589 \mathrm{meV}, E_{6}=382 \mathrm{meV}, E_{7}=544 \mathrm{meV}, E_{8}=549 \mathrm{meV}, E_{9}=577 \mathrm{meV}, E_{10}=534 \mathrm{meV}, E_{11}=589 \mathrm{meV}$, $E_{12}=576 \mathrm{meV}$. For all other barriers $E_{\mathrm{far}}=588 \mathrm{meV}$.

impurity atom. This takes into account the effect of the lattice stress induced by the tracer atom on the energy landscape observed by the vacancy. Each rate is simply proportional to the Boltzmann factor $\mathrm{e}^{-\Delta E / k_{\mathrm{B}} T}$, where $\Delta E$ is the activation energy for the considered diffusional move and $k_{\mathrm{B}} T$ is the thermal energy at temperature $T$. For the case of $\operatorname{In} / \mathrm{Cu}\left(\begin{array}{lll}0 & 0 & 1\end{array}\right)$ the diffusion barriers of the vacancy were calculated with the EAM method [46]. We used the interatomic potentials proposed by Finnis and Sinclair [47], where the metallic cohesion is taken into account via the second moment approximation to the tightbinding model. The calculation was done on a $15 \times 15 \times 15$ slab of copper. We computed the total energies of the system for a variety of different configurations of an In atom and a surface vacancy, every configuration being fully relaxed to a threshold force of $1.875 \times 10^{-12} \mathrm{~N}$. The barriers were obtained as the energy difference between the initial, relaxed configuration and the configuration with the moving atom at the point halfway between the stable sites. Figure 7 summarizes the values for the barriers. Since the barrier differences are large compared to $k_{\mathrm{B}} T$, the differences in jump probabilities are extremely large (see below for typical values).

When the vacancy reaches the perimeter of the lattice, its random walk is terminated, corresponding to the physical process of its recombination at steps on the surface. During its lifetime, the vacancy displaces atoms along its path, many of them multiple times. Thus, also the tracer atom can end up displaced from its original position at the time of recombination of the vacancy. Averaged over the random walks of many independent vacancies, this yields a probability distribution of the different displacement vectors that the tracer atom can make as a result of its (multiple) encounter with a single vacancy. Due to the boundary conditions, introduced by the finite size of the lattice and due to the distribution of exchange rates, an analytic solution to this problem is no longer possible.

When treating the above model numerically, we separate the motion of the vacancy and the tracer atom, as has been performed also in some of the analytical treatments referred to in Section 3.1. In our 
case of a finite lattice, this separation introduces an approximation, which is valid only if the tracer atom is relatively close to the middle of the lattice. First, we calculate the probabilities that the vacancy, released at one atomic spacing from the tracer, returns the first time to the tracer from equal $\left(p_{\text {eq }}\right)$, perpendicular $\left(p_{\text {perp }}\right)$ or opposite ( $\left.p_{\text {opp }}\right)$ directions; we also calculate the probability of its recombination $\left(p_{\text {rec }}\right)$ at the perimeter instead of returning to the tracer. Knowing these return and recombination probabilities, we calculate the statistics of the motion of the tracer atom, which performs a biased random walk of finite length. The probability distribution of the direction of each move with respect to the previous one, and the probability that a move was the last one, are obtained from the return and the recombination probabilities.

In practice, both the return probabilities of the vacancy and the displacement distribution of the tracer atom are obtained via direct evaluation of probabilities (enumeration of trajectories), which has better convergence properties than Monte-Carlo-type methods.

As an illustration of this enumeration method, we consider the computation of the vacancy return probabilities. We assign a variable to each lattice site, which measures the probability that the vacancy after $s$ atomic moves is at that site, while it has not exchanged with the tracer yet. Initially all probabilities are zero except at $(1,0)$ where it is unity. The vacancy starts there. The boundary acts as a trap for the vacancy, as does the origin (representing the tracer). For each atomic move of the vacancy we update all site variables in parallel by distributing the probability on each site to the four neighbors, according to the respective exchange probabilities. As the probability flows into the trap at the origin, we record the cumulative flow from each of the four directions leading to that site, which gives the return probabilities. This iteration converges quickly, and the convergence can be measured by the sum of the probabilities still on the lattice. The other computation, the motion of the tracer atom, is similar but slightly more complex. In that computation we assign a variable to each incoming edge of every site, which measures the probability that after $s$ moves - each corresponding to a vacancy return - the tracer is at the given site and that it arrived from the given direction. In addition, each site has a variable that accumulates the probability that the tracer becomes immobile at that site, meaning that the vacancy recombines when the tracer is at that site. These probabilities for the tracer arrival and immobilization are updated iteratively according to the previously obtained vacancy return probabilities.

To emphasize the importance of including the details of the tracer-vacancyinteraction, we mention that the fitted value of the room temperature recombination probability with no interaction is, $p_{\text {rec }}=0.027$. The value expected for $p_{\text {rec }}$, for the average step separation in the experiments of approximately 400 lattice spacings, is 0.23 - an order of magnitude larger. The theoretical dependence of $p_{\text {rec }}$ on the average terrace width is extremely weak (a logarithmic dependence was derived in a somewhat different context by Brummelhuis et al. [41]). In order to reach the low value of 0.027 , the average terrace width would have to be as large as $10^{25}$ atomic spacings, which is completely unphysical!

Using the EAM barriers, for typical parameters $T=320 \mathrm{~K}$ and $l=401$ the values for the return probabilities were $p_{\text {eq }}=1-2.4 \times 10^{-7}, p_{\text {perp }}=1.1 \times 10^{-7}, p_{\text {opp }}=4.2 \times 10^{-9}$, and the recombination probability $p_{\text {rec }}=1.1 \times 10^{-8}$. These values depend weakly on $l$ (e.g. the dependence of the mean square displacement, calculated later in this chapter from the return probabilities, is logarithmic: $\left\langle r^{2}\right\rangle \propto \log \left(l / l_{0}\right)$. This is a consequence of the fact that 2 is the marginal dimension for the return problem of the random walker. In higher-dimensional space the vacancy does not necessarily return and the return probability is asymptotically independent of the lattice size [43].

Again focusing on the case of an indium impurity in a $\mathrm{Cu}(001)$ lattice, we see that the diffusion barrier for a vacancy exchange with the indium atom is considerably lower than all other barriers. Therefore, in most cases the vacancy returns from the direction of its previous departure, and the individual moves of the tracer atom are strongly anti-correlated leading to no net displacement. Both the numerical and the theoretical treatment are simplified significantly if we do not have to follow the large number of ineffective "back and forth" exchanges of the vacancy and the tracer atom. For this purpose, consider the small probability $\varepsilon$ that the vacancy does not return from the same, equal direction. Thus $p_{\text {eq }}=1-\varepsilon$. In the case of indium in $\mathrm{Cu}(001)$ the EAM calculations yield 


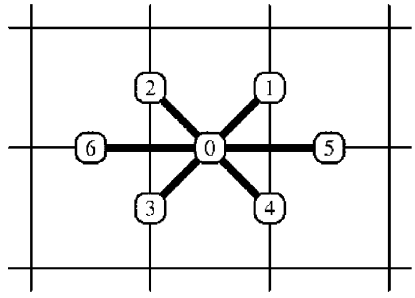

(a) (b)

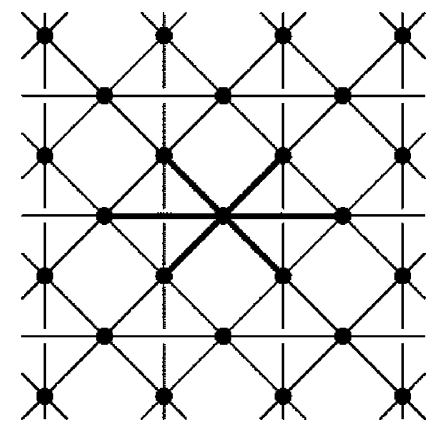

Figure 8: (a) The bond-to-bond moves of the vacancy-tracer pair. If we view the vacancy-tracer pair to live on the center of each bond, indicated by the labels, then the pair hops between these sites. The atomic lattice is drawn with thin lines. (b) The lattice (made of bonds of the atomic lattice) on which the vacancy-tracer pair walks. The neighbors that can be reached by a single move from the site at the center are shown with thick lines. This lattice can be considered as a square lattice, rotated by $45^{\circ}$ with respect to the atomic lattice, with some extra diagonal bonds. Note that this is not a Bravais lattice.

$\varepsilon=2.4 \times 10^{-7}$ (see above). We now define

$$
\begin{aligned}
& \hat{p}_{\text {perp }}=\frac{p_{\text {perp }}}{\varepsilon} \\
& \hat{p}_{\text {opp }}=\frac{p_{\text {opp }}}{\varepsilon} \\
& \hat{p}_{\text {rec }}=\frac{p_{\text {rec }}}{\varepsilon}
\end{aligned}
$$

and have

$$
2 \hat{p}_{\text {perp }}+\hat{p}_{\text {opp }}+\hat{p}_{\text {rec }}=1 \text {. }
$$

If we represent the quasi-bound state of the rapidly exchanging (on average $1 / \varepsilon$ times) vacancy and impurity atom with the position of a bond of the original lattice, then the vacancy-tracer pair walks on the bonds of the original lattice. The pair moves to each of the four perpendicular bonds with probability $\hat{p}_{\text {opp }} / 2$, and to each of the two parallel ones with $\hat{p}_{\text {opp }} / 2$, see Fig. 8 . (The factors $1 / 2$ reflect the probability for the vacancy to escape either at the right or the left side of the quasi-bound position, which is $1 / 2$ for both sides in the limit of vanishing $\varepsilon$. The advantage of this approach is twofold: the path of the tracer is made of fewer moves (beneficial for numerics), and the bond-to-bond moves are now independent (beneficial for theoretical treatment).

Using this, the tracer atom is described as if it forms a pair with the vacancy on one of the bonds adjacent to its original site, it walks on the bond lattice, and at the end of the walk (which happens after each move with probability $\hat{p}_{\text {rec }}$ ) it is released with equal probability at either end of the last visited bond. Results for the probabilities of the different jump lengths (beginning-to-end vectors of these trajectories) are shown in Fig. 9. Note, that the model calculations in Fig. 9 contain no adjustable parameters.

A general advantage of numerical modeling is that we have access to quantities which are difficult or impossible to measure experimentally. One example in our calculation is the probability that a tracer atom had an encounter with a vacancy, but its net displacement was zero. Although this value is non-zero, its temperature dependence is weak, which means that it can be incorporated in the constant jump-rate prefactor. This justifies the simplifying approach in the analysis of the experimental measurements to associate In-vacancy encounters with detectable (non-zero) jumps of the indium atom.

For a given set of diffusion barriers, our model has two parameters: the temperature and the lattice size. When we compare our theoretical results with experiments, both are known, and in principle there are no adjustable parameters. For example, in the case of $T=320 \mathrm{~K}$ (Fig. 9) - using the distance 


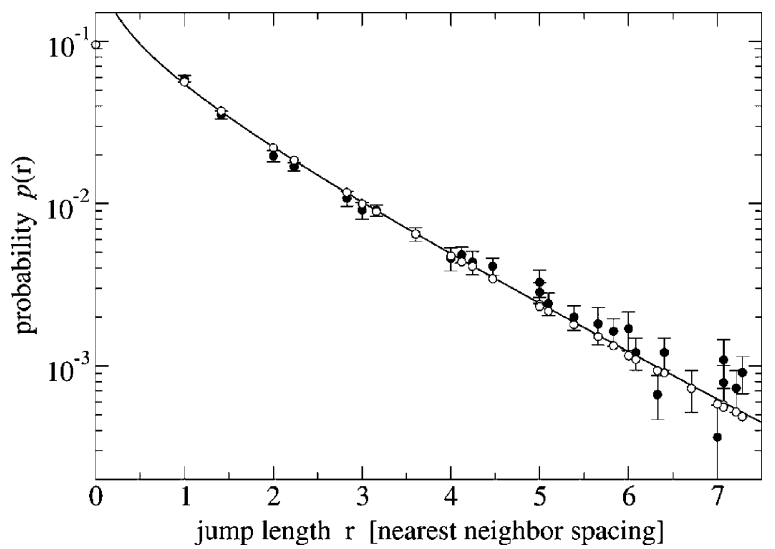

Figure 9: The probabilities of the jump lengths of the tracer atom for $T=320 \mathrm{~K}$ and $l=401$ lattice spacings. Filled circles correspond to experimental values (measured at this temperature and terrace size), open circles are from the model described in the text, and the solid curve is the continuum solution described in Section 3.3. The distribution depends only on the magnitude of the jump lengths with no directional dependence. (Each dataset is normalized separately such that the probabilities corresponding to a subset of the jump vectors, $1=|\boldsymbol{r}|=6$, add up to unity. These are the probabilities that are determined with good accuracy in the experiment.)

to the nearest step in the experiment as lattice size in the numerical model - the theory produced a good match with the experiment, but for the measurements at other temperatures we had to adjust the lattice size to obtain good agreement. Although the best fit lattice size in some cases was a factor of 2-3 smaller than the measured distance to the nearest step, the error that would otherwise occur in the return probabilities was much smaller, as these probabilities depend logarithmically on the lattice size. Undetected defects - acting as traps for the vacancies - closer to the In atom than the nearest step might account for these small discrepancies.

\subsection{Continuum model for tracer diffusion on a finite surface}

In the previous section we numerically solved the version of the tracer diffusion problem which was relevant for the $\mathrm{In} / \mathrm{Cu}\left(\begin{array}{l}0 \\ 0\end{array}\right)$ experiments. Although this already enables full comparison, the numerical solution has the disadvantage that it cannot be described with only a few parameters. In this section we develop a simple continuum description, where the overall shape of the jump length distribution is described with a single parameter.

We use our previous results for the return and recombination probabilities of the vacancy, and consider the random walk of the tracer-vacancy pair on the bond lattice. Let $?(\boldsymbol{r}, n)$ denote the probability that the Pl. provide tracer-vacancy pair is at position $\boldsymbol{r}$ at instance $n$, where $n$ counts the number of moves the tracer-vacancy ${ }^{\text {missing }}$ pair has already made. Since the subsequent moves of the pair are independent, we can write an effective denoted by "? diffusion equation for the evolution of ? $(\boldsymbol{r}, n)$ :

$$
\frac{\partial ?(\boldsymbol{r}, n)}{\partial n}=D_{\mathrm{eff}} \nabla^{2} ?-c ?
$$

The first term on the right hand side corresponds to the moves the pair makes on the bond lattice, here $D_{\text {eff }}$ denotes the mean square displacement per move of the pair. The second term corresponds to the recombination of the vacancy. ${ }^{1}$ When this occurs the pair breaks up. In the continuum approximation for space and $n$, the solution for a Dirac-delta initial condition at the origin is

$$
?(\boldsymbol{r}, n)=\frac{1}{4 \pi D_{\text {eff }} n} \exp \left(-\frac{r^{2}}{4 D_{\text {eff }} n}-c n\right)
$$


The final distribution of the tracer atom after the vacancy has recombined is obtained by the integration of the loss term in Eq. (4):

$$
p(\boldsymbol{r})=\int_{0}^{\infty} c ?(\boldsymbol{r}, n) n=\frac{1}{2 \pi} \frac{c}{D_{\mathrm{eff}}} K_{0}\left(\frac{r}{\sqrt{D_{\mathrm{eff}} / c}}\right),
$$

where $K_{0}$ is the modified Bessel function of order 0 . The functional form of this solution is similar to that of the infinite lattice system in Section 3.1, in spite of the differences introduced by the finite vacancy lifetime and the different boundary conditions.

The mean square displacement $\left\langle r^{2}\right\rangle$ is directly proportional to the square of the width of the Bessel solution. We can determine the width of the Bessel solution from the parameters $D_{\text {eff }}$ and $c$, which can be obtained from the return probabilities [33]

$$
\left\langle r^{2}\right\rangle \propto \frac{D_{\text {eff }}}{c}=\frac{\hat{p}_{\text {perp }}+\hat{p}_{\text {opp }}}{4 \hat{p}_{\text {rec }}} .
$$

This continuum solution is shown in Fig. 9. It closely follows the numerical solution of the model, even for relatively small distances from the origin, where one would expect stronger deviations between the discrete and continuum models.

\section{SOURCES AND SINKS OF SURFACE VACANCIES}

At temperatures close to RT, on most surfaces, it is well established that steps are the sole sources and sinks for adatoms [6]. Although never experimentally proven, the same can be expected for surface vacancies. In fact, this assumption was already explicit in the development of the numerical model that we presented in the previous section. In this section, we review data that directly supports this idea, again limiting ourselves to the case of $\mathrm{In} / \mathrm{Cu}(001)$. This data justifies the approach that was used in the previous section.

If we assume that the steps are indeed the sources and sinks for surface vacancies and we confine ourselves to the simplest case where there is no interaction between the vacancy and the tracer atom, the recombination probability of a vacancy, $p_{\text {rec }}$, introduced in the previous section, will decrease with increasing distance from a step. This is schematically illustrated in Fig. 10. This decrease in $p_{\text {rec }}$ with distance from a step allows us to experimentally verify whether the steps are indeed the sole sources and sinks for vacancies. The experimental verification consists of the following. Assume that we are tracking the motion of an embedded atom somewhere in a terrace, a given distance away from a step. Once a vacancy has formed at the step, has diffused to the embedded atom, and has had an initial exchange with the atom (i.e. in our measurements we observe the embedded atom to make a jump), the probability for it to have further encounters with the same vacancy is determined by the value $1-p_{\text {rec }}$. Since $p_{\text {rec }}$ decreases with increasing distance to the step, the vacancy will on average have more encounters with the tracer atom the further it is away from the step. This will cause the atom to be displaced over a larger distance. In fact, in our theoretical treatment we already found that the average jump length should increase logarithmically with increasing distance from the step.

For the case of $\mathrm{In} / \mathrm{Cu}(001)$ we performed several measurements of the average length of the long jumps of the indium atoms for several different distances from a step. The results are depicted in Fig. 11.

Our measurements indeed show the expected logarithmic increase in mean square jump length with increasing distance away from a step, and provide confirmation that steps are indeed the sole sources and sinks for surface vacancies. Having established the role of the steps as sources and sinks for vacancies we speculate on how the vacancies are formed at the steps. One can envision several different mechanisms at steps that lead to the formation of a surface vacancy. All of them involve removing an atom from a kink, a step or (least likely) from the terrace itself. Since the attachment and detachment of atoms from kinks is often the energetically least costly way to detach an atom from a step, this appears to be the most likely initial process in the formation of a surface 
vacancy. Calculations on this problem have been performed in the context of the observations on $\mathrm{Mn} / \mathrm{Cu}\left(\begin{array}{l}0 \\ 0\end{array}\right)$ that were mentioned previously [48-50]. In these calculations it was indeed found that the formation of step vacancies at kinks and the subsequent diffusion of these vacancies along a step and their release into the terrace is the most likely scenario for the creation of surface vacancies in a terrace.

(b)

(a)

(c)

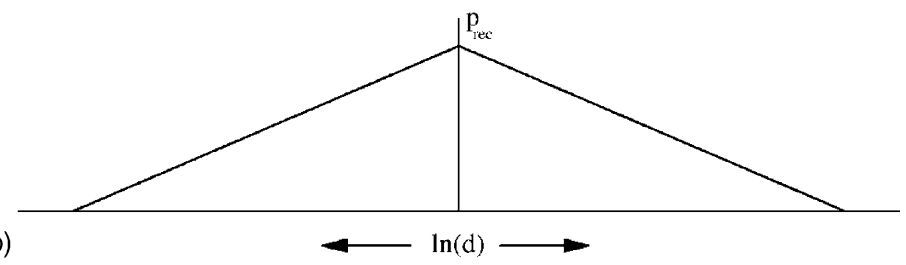

Figure 10: A schematic illustration of the effect of the presence of a step on the diffusion of a surface vacancy. (a) Schematic topography, with a step in the middle. (b) The recombination probability depends logarithmically on the distance. (c) Random walks that bring the vacancy far from the step will result on average in a much larger number of encounters with a tracer atom on the terrace than shorter random walks.

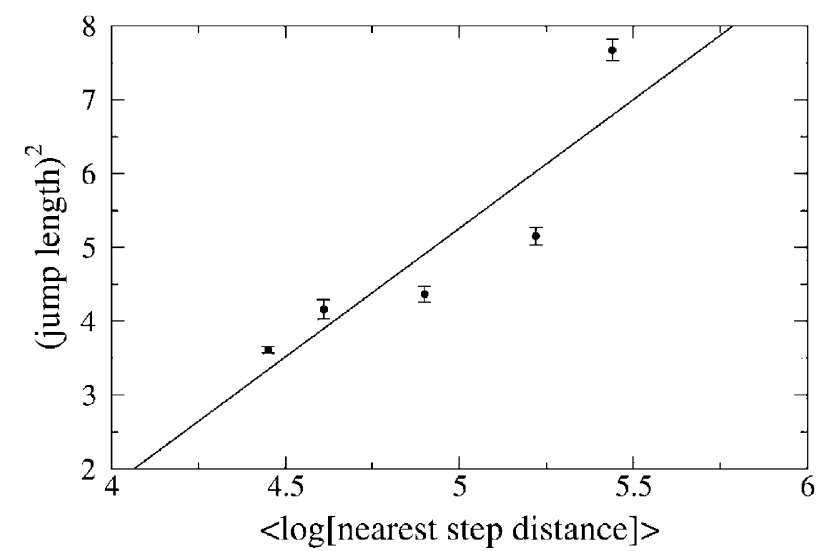

Figure 11: Plot of the mean square jump length versus the average logarithm of the distance of the measurement area to the steps. The graph shows that measurements that are made further away from a step yield larger jump lengths. All distances were measured in atomic spacings. 


\section{VACANCY ENERGETICS}

\subsection{Activation energy for vacancy-mediated tracer diffusion}

In this section, we discuss the energetics of the vacancy-mediated diffusion of tracer atoms in a surface. In particular, we focus on the energetics and the profound differences that are encountered in interpreting

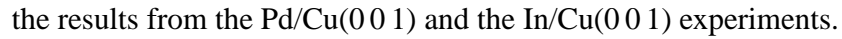

Ignoring the effect of the proximity of steps, for both systems it was found that the jump length distribution is independent of temperature over the temperature interval from 304 to $342 \mathrm{~K}$. Temperature dependent measurements of the rate of long jumps of the In and Pd atoms were performed over the same temperature interval. The results for both systems are plotted in Fig. 12.

Both graphs convincingly show Arrhenius behavior. The activation energies that are extracted from the two graphs, however, differ from each other by more than the statistical error margins. In the next section we will discuss this difference in more detail.

We should mention here that a better way to extract the activation energies for vacancy-mediated diffusion would be to plot the tracer diffusion coefficient of the embedded atoms vs. $1 / k T$, rather than their jump rate. As we discussed in Section 4, the mean square jump length depends on the proximity of steps, and so does the average jump frequency. This adds non-statistical noise to the two plots in Fig. 12. However, it can be shown easily that these effects on jump length and jump rate cancel in the resulting tracer diffusion coefficient, which thus becomes independent of the distance to steps. In this way, a more accurate value for the activation energy has been obtained for the case of $\operatorname{In} / \mathrm{Cu}(001)$ of $717 \pm 30 \mathrm{meV}[23]$.

\subsection{Interpretation of the energetics}

By definition, the rate at which the tracer atom is displaced by a surface vacancy is the product of the vacancy density at the site next to the tracer times the rate at which vacancies exchange with the tracer atom. For the case where the interaction between the tracer atom and the vacancy is negligible, the activation energy obtained from the temperature dependence of the total displacement rate equals the sum of the vacancy formation energy $E_{\mathrm{F}}$ and the vacancy diffusion barrier $E_{\mathrm{D}}$. When the measurements are performed with finite temporal resolution and if there is an interaction present between the vacancy and the indium atom, this simple picture changes.

We assume that the time resolution of the STM-measurements is sufficient to discriminate the effect of the entire random walk of one individual vacancy from that of the next, which was already shown to be the case in Section 2.2. The starting situation is shown in Fig. 13. A tracer atom is embedded in the origin of a square lattice with a vacancy sitting next to it at $(1,0)$. We assume that the only diffusion barrier that is modified by the tracer atom is that for vacancy-tracer exchange.
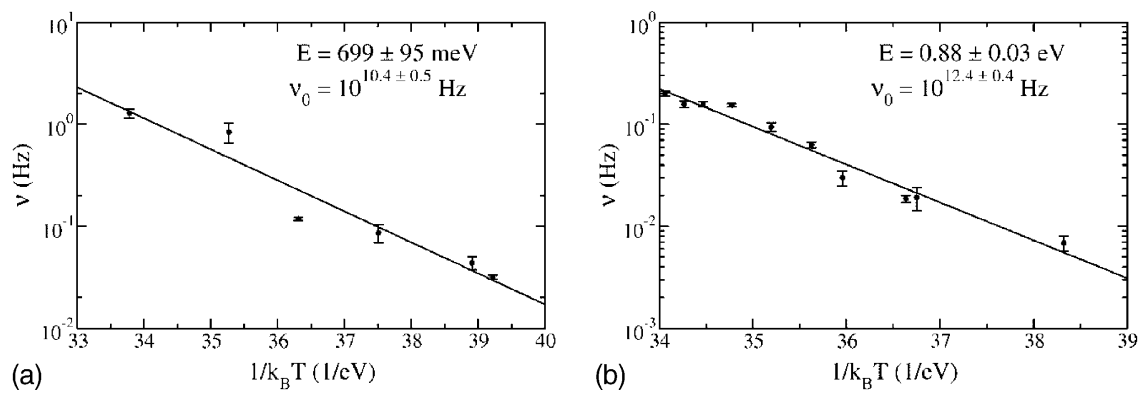

Figure 12: Rate of long jumps vs. $1 / k T$ for (a) In and (b) Pd. For both fits the obtained activation energies and prefactors are shown in the graph. 


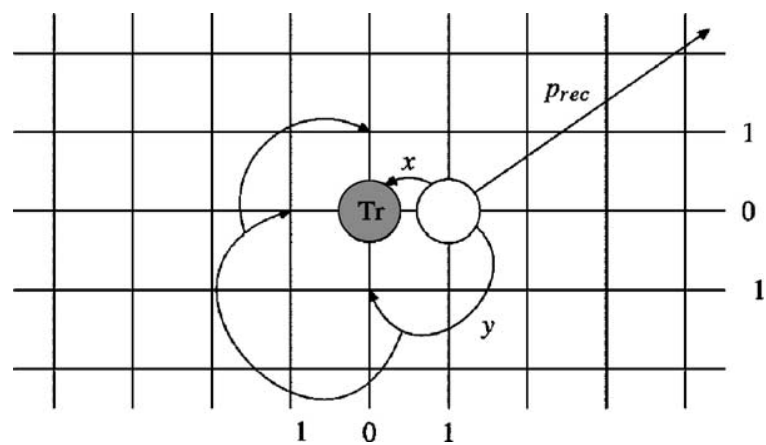

Figure 13: The random walk starting situation, with the vacancy directly next to the tracer atom. The probabilities $x, y$ and $p_{\text {rec }}$ are introduced in the text.

We now define the following additional quantities:

- $x$ : the probability that in the first move the vacancy immediately changes places with the tracer.

- $y$ : the probability that the vacancy does not immediately change places with the tracer but that it returns to any of the four sites neighboring the tracer.

- $p_{\text {rec }}$ : the recombination probability of the vacancy, i.e. the probability that the vacancy does not change places with the tracer anymore, but that it rather recombines at a step. This probability is determined by the distances to the nearby steps.

Note that $x+y+p_{\text {rec }}>1$, because, as is indicated in Fig. 13, there is the possibility that the vacancy returns one or more times to the sites neighboring the tracer atom and then recombines, without ever exchanging with the tracer. Only if one were to define the recombination probability as $p_{\text {rec }}^{\prime}$, the probability that the vacancy recombines without first returning to any of the sites neighboring the tracer atom, would $x+y+p_{\text {rec }}^{\prime}=1$. The rate at which jumps of the tracer are registered by the STM is determined by the rate at which total displacements with a length of at least one atomic spacing occur. Given the starting situation of Fig. 13, the probability that the tracer is displaced by the vacancy is given by

$$
p_{\text {dis }}=1-p_{\text {rec }}=x+y(x+y(x+y(\ldots)))=\sum_{N=0}^{\infty} y^{N} x=\frac{x}{1-y}
$$

The tracer atom is displaced if the vacancy returns to any of its 4 neighboring sites $0,1,2$, etc. times, and then exchanges places with it. The possibility that multiple displacements add up to a zero net displacement is ignored. ${ }^{2}$ The precise value of $x$, the probability to make a successful exchange, is determined by the energy difference between the modified $\left(E_{\mathrm{D}}^{\prime}\right)$ and unmodified $\left(E_{\mathrm{D}}\right)$ vacancy exchange barrier, $E_{\mathrm{D}}-E_{\mathrm{D}}^{\prime}$.

$$
x=x(T)=\frac{v_{0} \mathrm{e}^{-E_{\mathrm{D}}^{\prime} / k_{\mathrm{B}} T}}{v_{0} \mathrm{e}^{-E_{\mathrm{D}}^{\prime} / k_{\mathrm{B}} T}+3 v_{0} \mathrm{e}^{-E_{\mathrm{D}} / k_{\mathrm{B}} T}}=\frac{1}{1+3 \mathrm{e}^{-E_{\mathrm{D}}-E_{\mathrm{D}}^{\prime} / k_{\mathrm{B}} T}}
$$

where we assume the jumps in all four directions (unmodified and modified) to exhibit the same attempt frequency $n_{0}$. Examination of the random walk of the vacancy when it does not exchange with the tracer but returns to any of the neighboring sites, shows that only the first step of this random walk pathway depends on temperature and $y$ can therefore be split up into a temperature dependent and a temperature independent part

$$
y=y(T)=(1-x(T)) C
$$


where $0=C=1$ is a constant which is determined by the geometry of the lattice, and especially by the distribution of distances between the starting position of the vacancy and the absorbing boundaries (steps). It can be evaluated numerically. Substituting Eq. (10) in (8), the probability to have at least one displacement is equal to

$$
\begin{aligned}
p_{\text {dis }} & =\frac{x(T)}{1-y(T)} \\
& =\frac{x(T)}{1-(1-x(T)) C} \\
& =\frac{1}{1+3(1-C) \mathrm{e}^{-E_{\mathrm{D}}-E_{\mathrm{D}}^{\prime} / k_{\mathrm{B}} T}}
\end{aligned}
$$

From Eq. (11) it is clear that the final rate of long jumps will contain exponential terms not only in the numerator, but also in the denominator. The rate of long jumps should not show normal thermally activated Arrhenius behavior.

The observed rate of long jumps is equal to the equilibrium rate at which vacancies exchange with the tracer atom, divided by the average number of elementary displacements caused by a single vacancy, given that the vacancy has displaced the tracer atom at least once. This average number of displacements $\langle n\rangle$ is given by

$$
\begin{aligned}
\langle n\rangle & =1+0 p_{\text {rec }}+1\left(1-p_{\text {rec }}\right) p_{\text {rec }}+2\left(1-p_{\text {rec }}\right)^{2} p_{\text {rec }}+\cdots \\
& =\frac{1}{p_{\text {rec }}} \\
& =\frac{1}{1-C}\left(\frac{1}{1-x(T)}-C\right) \\
& =1+\frac{\mathrm{e}^{E_{\mathrm{D}}-E_{\mathrm{D}}^{\prime} / k_{\mathrm{B}} T}}{3(1-C)}
\end{aligned}
$$

Using Eq. (12), the observed rate of long jumps is equal to

$$
\nu_{\mathrm{LJ}}=v_{0} \frac{\mathrm{e}^{-\left(E_{\mathrm{F}}+E_{\mathrm{D}}+\Delta\right) / k_{\mathrm{B}} T}}{1+\mathrm{e}^{-\Delta / k_{\mathrm{B}} T} / C^{\prime}}
$$

where $E_{\mathrm{F}}$ is the vacancy formation energy, and

$$
\begin{aligned}
C^{\prime} & =3(1-C) \\
\Delta & =E_{\mathrm{D}}^{\prime}-E_{\mathrm{D}}
\end{aligned}
$$

In the case of no interaction, the tracer is "identical" to copper, so that $\Delta=0,\langle n\rangle$ becomes $4-3 c / 3$ $(1-C)$ and the rate of long jumps reduces to

$$
\nu_{\mathrm{LJ}}=\nu_{0} \frac{\mathrm{e}^{-\left(E_{\mathrm{F}}-E_{\mathrm{D}}\right) / k_{\mathrm{B}} T}}{1+\left(1 / C^{\prime}\right)}=\frac{v}{1+\left(1 / C^{\prime}\right)}
$$

where $v$ is the vacancy-induced jump rate of $\mathrm{Cu}$ atoms in the clean $\mathrm{Cu}(001)$ surface. The denominator corresponds to the average number $\langle n\rangle$ of exchanges contributing to one long jump.

In the extremely repulsive case $\Delta / k_{\mathrm{B}} T \gg 1$ and the average number of displacements $\langle n\rangle$ becomes approximately equal to 1 . Equation (13) reduces to

$$
\nu_{\mathrm{LJ}}=\nu_{0} \mathrm{e}^{-\left(E_{\mathrm{F}}-E_{\mathrm{D}}+\Delta\right) / k_{\mathrm{B}} T}=\nu_{0} \mathrm{e}^{-\left(E_{\mathrm{F}}-E_{\mathrm{D}}^{\prime}\right) k_{\mathrm{B}} T}
$$

In the extremely attractive case $\Delta / k_{\mathrm{B}} T \ll-1$ and the average number of jumps $\langle n\rangle$ is $1 /(1-$ $C)(1 / 3) \mathrm{e}^{-\Delta / k_{\mathrm{B}} T}$. Equation (13) now reduces to

$$
v_{\mathrm{LJ}}=3 v_{0}(1-C) \mathrm{e}^{-\left(E_{\mathrm{F}}-E_{\mathrm{D}}\right) / k_{\mathrm{B}} T}
$$


We immediately see the fundamental difference between attraction and repulsion. The apparent activation energy for strong attraction is identical to that for the copper surface itself, while it is larger in the case of repulsion. This asymmetry between attraction and repulsion is caused by the fact that for moderate to strong attraction, the probability $x$ rapidly approaches unity, meaning that the arrival of a vacancy next to the tracer is almost guaranteed to cause a (long) jump of the tracer. In contrast, in the case of repulsion, the probability $x$ scales with the Boltzmann-factor containing the exchange barrier of the vacancy and the embedded atom.

The difference between attraction and repulsion is also apparent in the context of the rate-limiting step. For the case of attraction, the long-jump rate is limited by how long it takes a vacancy to revisit the tracer once it escapes from the attractive potential. This rate is given by the diffusion of the vacancy among the copper atoms of the terrace. For the case of repulsion, on the other hand, the long-jump rate is limited by the vacancy overcoming the barrier to exchange with the tracer, presumably arriving at a nearest-neighbor site several times before doing so. EAM [33] and first-principles [24] calculations have shown that the exchange barrier for a vacancy in the $\mathrm{Cu}(0001)$ surface with an In or a Pd atom is $243 \mathrm{meV}$ and $466 \mathrm{meV}$ respectively. First-principles calculations [24] showed that the vacancy diffusion barrier in the $\mathrm{Cu}(001)$ surface is equal to $426 \mathrm{meV}$. For practical values of $1 / k_{\mathrm{B}} T$ the interpretation of the activation energies that we showed previously is now obvious. For indium we are in the limit $\Delta / k_{\mathrm{B}} T \ll-1$ and the measured activation energy is equal to the sum of the vacancy formation energy, $E_{\mathrm{F}}$, and the vacancy-Cu diffusion barrier, $E_{\mathrm{D}}$, whereas in the case of $\mathrm{Pd}$ we are in the case of moderate repulsion and the activation energy should be taken to be the sum of the vacancy formation energy and the vacancy-Pd exchange barrier. We mention again that the two energy values that make up the activation energy cannot be separately determined experimentally. In order to obtain their values an independent measurement of either one of them is needed.

\section{VACANCY-MEDIATED SELF-DIFFUSION}

The importance of the measurements that we have presented so far for the diffusion of embedded tracer atoms becomes evident when we now use these measurements and the model discussed in Section 3 to evaluate the "invisible" mobility of the $\mathrm{Cu}$ atoms in a $\mathrm{Cu}(001)$ terrace. The results presented in Section 2 imply that not just the tracer atom, but all atoms in the surface are continuously moving. From the tracer diffusion measurements of $\mathrm{In} / \mathrm{Cu}\left(\begin{array}{lll}0 & 0 & 1\end{array}\right)$ we have established that the sum of the vacancy formation energy and the vacancy diffusion barrier in the clean $\mathrm{Cu}\left(\begin{array}{ll}0 & 01\end{array}\right)$ surface is equal to $717 \mathrm{meV}$.

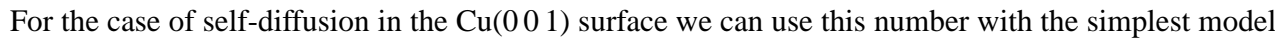
that we discussed in Section 3.2, i.e. all atoms are equal and no interaction between the vacancy and

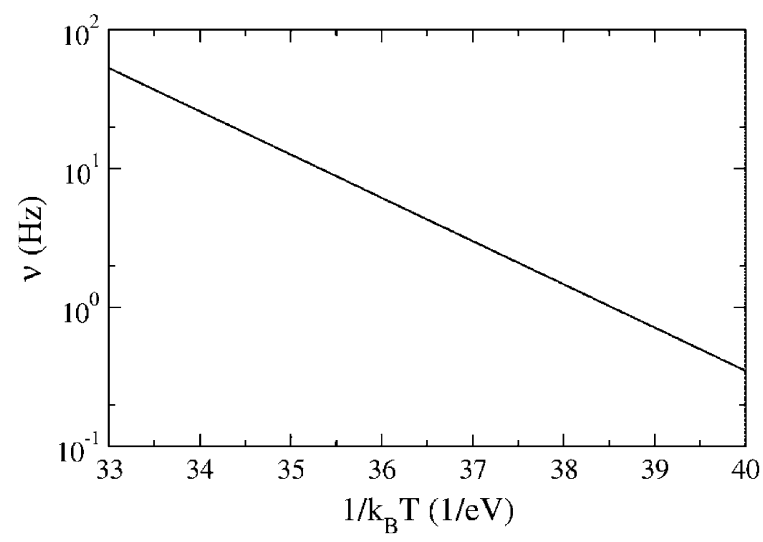


the tracer atom. In doing so we find a room temperature hop rate for the self-diffusion of $\mathrm{Cu}$ atoms in a $\mathrm{Cu}(001)$ terrace of $n=0.48 \sim \mathrm{s}^{-1}$. In other words, every terrace $\mathrm{Cu}$ atom is displaced by a vacancy, on average, about once per two seconds at room temperature and about 200 times $/ \mathrm{sec}$ at $100^{\circ} \mathrm{C}$. We illustrate this motion by plotting the calculated average displacement rate of $\mathrm{Cu}$ terrace atoms vs. $1 / \mathrm{kT}$ in Fig. 14.

\section{SUMMARY}

Surface vacancies were shown to be responsible for the motion of embedded In and Pd atoms in the $\mathrm{Cu}(001)$ surface. The density of surface vacancies at room temperature is extremely low, but they diffuse through the surface at an extremely high rate leading to significant diffusion rates of $\mathrm{Cu}\left(\begin{array}{lll}0 & 0 & 1\end{array}\right)$ terrace atoms. In the STM-measurements the rapid diffusion of these vacancies leads to long jumps of embedded tracer atoms. Measurements of the jump length distribution show a shape of the distribution that is consistent with the model that we discussed in Section 3. In turn, this shows that the vacancymediated diffusion process can be accurately described with the model that is presented in Section 3, provided that the interaction between the tracer atom and the surface vacancy is properly taken into account. The role of steps as sources and sinks for surface vacancies has been confirmed by measuring the position dependent jump length of embedded indium atoms. Near a step the jump rate of an indium atom is increased, but at the same time, given the shorter lifetime of vacancies near a step, the jump length is decreased. The effect of vacancy-tracer interaction was discussed for two different systems, $\mathrm{In} / \mathrm{Cu}\left(\begin{array}{lll}0 & 0 & 1\end{array}\right)$ (attractive interaction) and $\mathrm{Pd} / \mathrm{Cu}\left(\begin{array}{lll}0 & 0 & 1\end{array}\right)$ (repulsive interaction). The activation energies that are obtained for these two systems have to be interpreted differently.

The measurements that we discussed comprehensively show that the motion of vacancies through a surface leads to significant motion of atoms in the terrace. Despite the fact that this motion is often impossible to visualize, it is an important mechanism for mass transport of atoms within a terrace. Moreover, as we showed in this chapter for the case of copper, in the more open $\left(\begin{array}{lll}0 & 01\end{array}\right)$ surface it is most likely the dominant mechanism.

\section{NOTES}

1. Note that since $n$ counts the number of moves of the vacancy-tracer pair on the bond lattice, the tern $-\mathrm{c}$ ? does not imply that the vacancy can recombine at any lattice site - in fact it recombines at terrace steps, between subsequent returns to the In atom.

2. This is justified by numerical calculations presented elsewhere where this problem was addressed for the case of indium [33]. The calculations show that the fraction of jumps that have zero net length changes only marginally with temperature.

\section{ACKNOWLEDGMENTS}

Sandia National Laboratories is a multiprogram laboratory operated by Sandia Corporation, a Lockheed Martin Company, for the United States Department of Energy under contract DE-AC04-94AL85000. This work was supported in part by the Division of Materials Science and Engineering, Office of Science, U.S. Department of Energy. This work is part of the research program of the "Stichting voor Fundamenteel Onderzoek der Materieíi (FOM)" and is financially supported by the "Nederlandse Organisatie voor Wetenschappelijk Onderzoekíi (NWO)."

\section{REFERENCES}

[1] Müller E.W., J. App. Phys. 28 (1957) 1.

[2] Kellogg G.L., Surf. Sci. Rep. 21 (1994) 1, and references therein.

[3] Ehrlich G., in: Surface Diffusion: Atomistic and Collective Processes, M.C. Tringides, eds, NATO ASI Series B, Vol. 360, Plenum Press, New York, 1997, 23. 
[4] Binnig G., Rohrer H., Gerber C., Weibel E., Appl. Phys. Lett. 40 (1982) 178.

[5] Besenbacher F., Rep. Prog. Phys. 59 (1996) 1737, and references therein.

[6] Jeong H.-C., Williams E.D., Surf. Sci. Rep. 34 (1999) 171, and references therein.

[7] Giesen M., Prog. in Surf. Sci. 68 (2001) 1, and references therein.

[8] Poensgen M., Wolf J.F., Frohn J., Giesen M., Ibach H., Surf. Sci. 274 (1992) 430.

[9] Pimpinelli A., Villain J., Wolf D.E., Metois J.J., Heyraud J.C., Elkinani I., Uimin G., Surf. Sci. 295 (1993) 143.

[10] Kuipers L., Hoogeman M.S., Frenken J.W.M., Phys. Rev. Lett. 71 (1993) 3517.

[11] Morgenstern K., Rosenfeld G., Poelsema B., Comsa G., Phys. Rev. Lett. 74 (1995) 2058.

[12] Pearson C., Borovsky B., Krueger M., Curtis R., Ganz E., Phys. Rev. Lett. 74 (1995) 2710.

[13] Kuipers L., Hoogeman M.S., Frenken J.W.M., van Beijeren H., Phys. Rev. B 52 (1995) 11387.

[14] Giesen M., Icking-Konert G.S., Stapel D., Ibach H., Surf. Sci. 366 (1996) 229.

[15] Morgenstern K., Rosenfeld G., Lægsgaard E., Besenbacher F., Comsa G., Phys. Rev. Lett. 80 (1998) 556.

[16] Ehrlich G., Scanning Microscopy 4 (1990) 829.

[17] Ehrlich G., Hudda F.G., J. Chem. Phys. 44 (1966) 1039.

[18] Schwoebel R.L., Shipsey E.J., J. of Appl. Phys. 37 (1966) 3682.

[19] Kyuno K., Ehrlich G., Phys. Rev. Lett. 81 (1998) 5592.

[20] Flores T., Junghans M., Wuttig M., Surf. Sci. 371 (1997) 1.

[21] van Gastel R., Somfai E., van Saarloos W., Frenken J.W.M., Nature 408 (2000) 665.

[22] van Gastel R., Somfai E., van Albada S.B., van Saarloos W., Frenken J.W.M., Phys. Rev. Lett. 86 (2001) 1562.

[23] van Gastel R., Somfai E., van Albada S.B., van Saarloos W., Frenken J.W.M., Surf. Sci. 521 (2002) 10.

[24] Grant M.L., Swartzentruber B.S., Bartelt N.C., Hannon J.B., Phys. Rev. Lett. 86 (2001) 4588.

[25] McCarty K.F., Nobel J.A., Bartelt N.C., Nature 412 (2001) 622.

[26] Poelsema B., Hannon J.B., Bartelt N.C., Kellogg G.L., to be published.

[27] Stoltze P., J. Phys.: Cond. Matt. 6 (1994) 9495.

[28] van Gastel R., Ph.D. thesis, Universiteit Leiden, 2001.

[29] Kobayashi A., Grey F., Snyder E., Aono M., Surf. Sci. 291 (1993) L739.

[30] Molinàs-Mata P., Mayne A.J., Dujardin G., Phys. Rev. Lett. 80 (1998) 3101.

[31] Mayne A.J., Rose F., Bolis C., Dujardin G., Surf. Sci. 486 (2001) 226.

[32] Meyer G., Bartels L., Zöphel S., Henze E., Rieder K.-H., Phys. Rev. Lett. 78 (1997) 1512.

[33] Somfai E., van Gastel R., van Albada S.B., Frenken J.W.M., van Saarloos W., Surf. Sci. 521 (2002) 26.

[34] Hoogeman M.S., Glastra van Loon D., Loos R.W.M., Ficke H.G., de Haas E., van der Linden J.J., Zeijlemaker H., Kuipers L., Chang M.F., Klik M.A.J., Frenken J.W.M., Rev. Sci. Instr. 69 (1998) 2072.

[35] Swartzentruber B.S., Phys. Rev. Lett. 76 (1996) 459.

[36] Hannon J.B., Klünker C., Giesen M., Ibach H., Bartelt N.C., Hamilton J.C., Phys. Rev. Lett. 79 (1997) 2506.

[37] Boisvert G., Lewis L.J., Phys. Rev. B 56 (1997) 7643.

[38] van Gastel R., Rosu M.F., Rost M.J., Niesen L., Frenken J.W.M., submitted.

[39] Feller W., An introduction to probability theory and its applications, 3rd ed., Wiley \& Sons, New York, 1968, Vol. 1, 342-371.

[40] van Beijeren H., Kutner R., Phys. Rev. Lett. 55 (1985) 238.

[41] Brummelhuis M.J.A.M., Hilhorst H.J., J. Stat. Phys. 53 (1988) 249.

[42] Toroczkai Z., Int. J. Mod. Phys. B 11 (1997) 3343.

[43] Newman T.J., Phys. Rev. B 59 (1999) 13754.

[44] Bénichou O., Oshanin G., Phys. Rev. E 64 (2001) 020103(R).

[45] Abramowitz M., Stegun I.A., Handbook of Mathematical Functions: with formulas, graphs, and mathematical tables, Dover, New York, 1972.

[46] Daw M.S., Baskes M.I., Phys. Rev. B 29 (1984) 6443.

[47] Finnis M.W., Sinclair J.E., Philos. Mag. A 50 (1984) 45.

[48] Tréglia G., Legrand B., Saúl A., Flores T., Wuttig M., Surf. Sci. 352 (1996) 552.

[49] Ibach H., Giesen M., Flores T., Wuttig M., Treglia G., Surf. Sci. 364 (1996) 453.

[50] Flores T., Junghans S., Wuttig M., Surf. Sci. 371 (1997) 14. 